\title{
COMPRESSIVE SENSING BASED TARGET COUNTING AND LOCALIZATION EXPLOITING JOINT SPARSITY
}

\author{
Eva Lagunas, Shree Krishna Sharma, Symeon Chatzinotas and Björn Ottersten \\ Interdisciplinary Centre for Security, Reliability and Trust (SnT) \\ University of Luxembourg, 4 rue Alphonse Weicker, L-2721, Luxembourg \\ Email: \{eva.lagunas,shree.sharma,symeon.chatzinotas,bjorn.ottersten\}@uni.lu
}

\begin{abstract}
One of the fundamental issues in Wireless Sensor Networks (WSN) is to count and localize multiple targets accurately. In this context, there has been an increasing interest in the literature in using Compressive Sensing (CS) based techniques by exploiting the sparse nature of spatially distributed targets within the monitored area. However, most existing works aim to count and localize the sparse targets utilizing a Single Measurement Vector (SMV) model. In this paper, we consider the problem of counting and localizing multiple targets exploiting the joint sparsity feature of a Multiple Measurement Vector (MMV) model. Furthermore, the conventional MMV formulation in which the same measurement matrix is used for all sensors is not valid any more in practical time-varying wireless environments. To overcome this issue, we reformulate the MMV problem into a conventional SMV in which MMVs are vectorized. Subsequently, we propose a novel reconstruction algorithm which does not need the prior knowledge of the sparsity level unlike the most existing CS-based approaches. Finally, we evaluate the performance of the proposed algorithm and demonstrate the superiority of the proposed MMV approach over its SMV counterpart in terms of target counting and localization accuracies.
\end{abstract}

Index Terms - Wireless sensor networks, target counting, target localization, compressive sensing.

\section{INTRODUCTION}

Wireless Sensor Networks (WSNs), in which several sensor nodes equipped with sensing/actuation devices collaboratively perform a common task, have received important attention in several applications such as environmental monitoring, intrusion detection, robust target tracking and localization, and radio environment map construction [1]. In most of the aforementioned applications, target counting is often considered as the first step and the problem of counting multiple targets is non-trivial since it needs to differentiate and count the targets [2]. In addition, determining the physical location of targets such as wireless transmit nodes is a fundamental requirement and a key challenge in WSN applications. In this context, target counting and localization has been a topic of active research in the recent years [2-5].

Compressive Sensing (CS), a signal processing technique which can recover sparse signals utilizing far fewer samples or measurements than those required by the conventional non-CS based methods [6-8], has recently received important attention in solving various problems in WSNs in addition to its applications in several other areas such as digital image processing, radar imaging, Cognitive Radio (CR) $[9,10]$, etc. The application of CS in many WSN applications has been mainly motivated due to the following aspects [11]: (i) there may be a very limited number of active sensors as compared to the total number of sensors in the network, (ii) potential targets cover a small part of the total discrete spatial domain, which allows the localization problem to be linearized over a set of possible locations. In this context, this paper focuses on the application of the CS principle in WSNs in order to count and localize the sparsely distributed targets.

1) Related Work: Received Signal Strength (RSS) is one of the most widely accepted and used parameters for localization in WSNs due to the fact that it can be applied to almost any radio device. Moreover, it is by far the cheapest and the simplest option. Examples of target localization by interpreting RSS can be found in [12-14]. The existing target counting and localization methods can be broadly categorized into the following types [2]: (i) non-CS based, and (ii) CS-based. The non-CS based target counting methods can be further categorized into: (i) Minimalistic approach [15], (ii) Binary sensingbased approach [16,17], and (iii) Clustering-based approach [18,19]. In the first approach, each sensor outputs the number of distinct targets within its sensing region and in the second approach, the number of targets are estimated by assuming that a sensor reports a value 1 if one or more targets are detected within its sensing region, and 0 , otherwise. The third approach identifies multiple non-overlapping clusters consisting of one or more targets.

In order to take advantage of the sparse nature of WSNs, recent contributions have focussed on CS-based target counting and localization $[2,4,5,11,20-24]$. Out of these contributions, [2,11] have focussed on CS-based target counting, [4,5] has focussed on both target counting and localization, and the rest have focussed on CSbased localization. The contribution in [11] has proposed CS-based Baysian detection and heuristic approaches which can reduce the sampling rate by $25 \%$ without compromising the detection performance. Furthermore, authors in [2] propose a novel Greedy Matching Pursuit (GMP) algorithm utilizing the CS based approach for sparse target counting and localization in WSNs and prove that the proposed GMP algorithm can accurately recover a sparse signal with a high probability. Besides, the contribution in [4] applies CS theory for device-free counting and localization in order to count and localize multiple targets in sensor networks utilizing a GMP algorithm based on the RSS measurements.

Multiple Measurement Vectors (MMV) in a CS framework have been shown to further reduce the required number of samples [25-27], and can provide substantial improvements on the localization accuracy given the implicit sparse representation of the problem. Unlike SMV, the set of observations in the MMV model is represented as a set of jointly sparse vectors, represented as a matrix, sharing their common non-zero supports, i.e., the physical location of the targets to be localized. In other words, all the employed 
sensors observe the targets at the same positions. This feature is also known as joint sparsity in the general CS literature [28-30].

2) Contributions: In this paper, we focus on counting and localizing multiple targets using a small number of RSS measurements collected by a few sensors in contrast to the traditional non-CS based approach which requires a large number of sensors within the monitored area $[18,19]$. Most of the existing CS-based counting and localization techniques consider a Single Measurement Vectors (SMV) model, in which the observation/measurement is processed as a vector $[2,4]$. This is appropriate for WSN applications in which each of the sensors captures a single snapshot about the positions of the sparsely distributed targets $[2,23,24]$. However, in practice, a sensor may acquire multiple snapshots either exploiting the time [27] or the spatial diversity [31] of the problem, thus resulting in an MMV model. Motivated by this aspect, we apply an MMV-based CS approach in order to improve the target counting and localization accuracies. In particular, we extend the problem formulation presented in [2] to the MMV case. In [2], an SMV-based problem formulation was proposed together with an iterative reconstruction algorithm which can count and localize targets without the knowledge of the sparsity level. Here, we go a step further and consider the availability of multiple RSS measurement vectors at each of the sensors.

However, the conventional MMV formulation in which the same sparse snapshot is measured assuming the same measurement matrix is not valid any more since the channel realization might change from one time instant to another. Neither the joint sparsity models proposed in [30] fit in the proposed context, in which the sparsity is assumed to be time-varying. To overcome these issues, we reformulate the MMV into a conventional SMV in which the MMVs are vectorized. Based on the algorithm proposed in [2], we propose a novel GMP algorithm, particularly designed for the case where MMVs are available, to recover both the physical location and the exact number of targets present in the considered area. Unlike many existing sparse signal recovery algorithms, the proposed algorithm does not require the prior knowledge of the sparsity level nor any information about the noise component of the measurements.

3) Organization: The rest of the paper is organized as follows: Section II reviews the SMV-based target counting and localization method presented in [2]. Section III deals with the MMV-based target counting and localization problem by providing a mathematical formulation and the proposed GMP algorithm. Supporting simulation results are presented in Section IV, and finally, concluding remarks are provided in Section V.

\section{SMV-BASED TARGET COUNTING AND LOCALIZATION}

The goal of the proposed target counting and localization technique is to localize and count blind wireless nodes using the measured RSS captured at a few sensor positions. In this section, we review the SMV-based approach for target counting and localization presented in [2].

Let us assume the case in which the area of interest is divided into a discrete grid with $N$ points. Let $s_{i}$ be the number of targets at the $i$-th grid point, where $s_{i} \in\{0,1,2, \ldots, m\}$, with $m$ denoting the maximum number of targets that can be present in a single grid point. Based on the point-target model, the RSS observed by a sensor at location $j$ is given by,

$$
x_{j}=P_{0} g_{j, j} s_{j}+\sum_{\substack{i=1 \\ i \neq j}}^{N} P_{0} \frac{g_{i, j}}{d_{i, j}^{\alpha}} s_{i}+n_{j},
$$

where $P_{0}$ denotes the signal strength at the $i$-th grid point, $d_{i, j}$ denotes the distance between the $i$-th grid point and the $j$-th location, $g_{i, j}$ denotes the channel coefficient, which is assumed to follow a complex Gaussian distribution, and $n_{j}$ is the additive Gaussian white noise. The decay factor is denoted by $\alpha \in[2,5]$, whose value depends on the propagation environment.

Assuming that one sensor is placed at each grid, we have $N$ different RSS measurements, $x_{j}, j=1, \ldots, N$, which can be lexicographically ordered into a column vector $\mathbf{x}=\left[x_{1}, x_{2}, \ldots, x_{N}\right]^{T}$. In a similar way, stacking the elements $s_{i}, i=1, \ldots, N$ into a column vector, we can obtain the equivalent measurement model,

$$
\mathbf{x}=\mathbf{\Psi}+\mathbf{n},
$$

with $\mathbf{s}=\left[s_{1}, s_{2}, \ldots, s_{N}\right]^{T}, \mathbf{n}=\left[n_{1}, n_{2}, \ldots, n_{N}\right]^{T}$ and $\boldsymbol{\Psi}$ being an $N \times N$ matrix defined as,

$$
\Psi=P_{0}\left[\begin{array}{cccc}
1 & \frac{g_{2,1}}{d_{2,1}^{\alpha}} & \ldots & \frac{g_{N, 1}}{d_{N, 1}^{\alpha}} \\
\frac{g_{1,2}}{d_{1,2}^{\alpha}} & 1 & \ldots & \frac{g_{N, 2}^{\alpha}}{d_{N, 2}^{\alpha}} \\
\vdots & \vdots & \ddots & \vdots \\
\frac{g_{1, N}}{d_{1, N}^{\alpha}} & \frac{g_{2, N}}{d_{2, N}^{\alpha}} & \cdots & 1
\end{array}\right]
$$

Assuming that only $k$ out of $N$ grid points $(k \ll N)$ are occupied by targets, then target counting and localization problem can be reformulated as a CS problem. In particular, the $k$-sparse vector $\mathbf{s}$ can be recovered with only $M=O(k \log N)$ sensor measurements, where $k<M \ll N$. In other words, $M$ arbitrary sensors can be randomly deployed over the set of the grid center points, at most one per grid, and their RSS measurements suffice to recover $\mathbf{s}$ with high probability. Towards the objective of reducing the number of sensors, consider $\mathbf{y} \in \mathbb{R}^{M \times 1}$ consisting of elements randomly chosen from $\mathrm{x}$ as follows,

$$
\mathbf{y}=\mathbf{\Phi} \mathbf{x},
$$

where $\boldsymbol{\Phi}$ is the measurement matrix constructed by randomly selecting $M$ rows of an $N \times N$ identity matrix. Note that the element $\boldsymbol{\Phi}(m, j)=1$ indicates that the $m$-th sensor is located at the $j$-th grid point.

\section{MMV-BASED TARGET COUNTING AND LOCALIZATION}

In this paper, we assume the availability of MMV. In so doing, it is expected that the proposed method will achieve better performance compared to [2], since more information or constraints are considered in the solution. In this section, we first provide the mathematical formulation of the MMV-based localization problem. Subsequently, we propose a novel GMP algorithm suited for the joint sparsity MMV model which can count and locate the sparsely distributed targets accurately.

\subsection{Signal Model}

Let us consider that each sensor is able to capture $L$ snapshots of $N$ different RSS measurements at different time instants. The targets located within the monitored area are assumed to be static during 
the sensing time and thus, the spatial sparsity profile of the target locations is preserved. Therefore, unlike the SMV model presented in [2], $L$ multiple measurement vectors are available in our MMV model, which can be expressed as,

$$
\mathbf{x}_{l}=\Psi_{l} \mathbf{s}+\mathbf{n}_{l}, \quad \text { for } l=1, \ldots, L .
$$

Note that the channel conditions as well as noise might change with time but the sparse vector $\mathbf{s}$ remains unaltered. This scenario is different from the conventional MMV framework [25,26], in which the matrix $\Psi$ is common for each of the available measurements. As a consequence, conventional MMV theory and conventional MMVbased reconstruction algorithms do not apply to our problem.

For the sake of convenience, let us arrange the $L$ measurement vectors into a large column vector $\mathbf{X} \in \mathbb{C}^{N L \times 1}$ as follows,

$$
\mathbf{X}=\left[\begin{array}{llll}
\mathbf{x}_{1}^{T} & \mathbf{x}_{2}^{T} & \cdots & \mathbf{x}_{L}^{T}
\end{array}\right]^{T} .
$$

Since $\mathbf{s}$ is common to each of the measurement vectors, the sparse location model can be rewritten as,

$$
\mathbf{X}=\mathbf{G S}
$$

where $\mathbf{S} \in \mathbb{R}^{N L \times 1}$ is a vector formed by the concatenation of $L$ times the sparse vector $\mathbf{s}$, i.e., $\mathbf{S}=\mathbf{s} \otimes \mathbf{1}_{L}$ with $\otimes$ being the Kronecker product and $\mathbf{1}_{L}$ being an $L$-element column vector with all its elements equal to one. Matrix $\mathbf{G} \in \mathbb{R}^{N L \times N L}$ in (7) is given by,

$$
\mathbf{G}=\left[\begin{array}{cccc}
\boldsymbol{\Psi}_{1} & \mathbf{0} & \cdots & \mathbf{0} \\
\mathbf{0} & \boldsymbol{\Psi}_{2} & \cdots & \mathbf{0} \\
\vdots & \vdots & \ddots & \vdots \\
\mathbf{0} & \mathbf{0} & \cdots & \boldsymbol{\Psi}_{L}
\end{array}\right]
$$

where the sub-index $l$ in $\boldsymbol{\Psi}_{l}$ indicated different channel realizations and therefore, different realizations of the $\Psi$ matrix. Then, considering only the measurements captured at the reduced set of sensors, we have,

$$
\mathbf{Y}=\mathbf{\Omega X}=\mathbf{A S},
$$

where $\boldsymbol{\Omega}=\boldsymbol{\Phi} \otimes \mathbf{I}_{L}$, with $\mathbf{I}_{L}$ being an $L \times L$ identity matrix, and $\mathbf{A}=\mathbf{\Omega G} \in \mathbb{R}^{M L \times N L}$.

\subsection{Proposed Greedy Matching Pursuit Algorithm}

There are many CS reconstruction algorithms available in the literature to recover the sparse scene $\mathbf{S}$ from the reduced set of measurements $\mathbf{Y}$, most of them based on $l_{1}$-norm minimization [6]. However, the sparsity level $k$ and/or general prior knowledge of the error variance is commonly assumed to be known in most of the recovery algorithms. Here, we proposed a novel GMP algorithm based on the principles of the one presented in [2], which provides an estimate of the value of $k$ and an estimate of the locations and values of the non-zero entries in the sparse signal s. Moreover, unlike [2], the proposed algorithm is able to take advantages of the MMV model and its joint sparsity feature. The proposed GMP algorithm exploiting the joint sparsity of the MMV model (9) is described with detail in Algorithm 1. Essentially, at each iteration, the algorithm selects the grid point and the number of targets it contains that contribute the most to the received measurements. Then its contribution is subtracted off and the algorithm iterates on the residual. The algorithm is halted when the number of targets corresponding to the selected grid is zero, assuming that the algorithm has already identified the correct set of grid points.

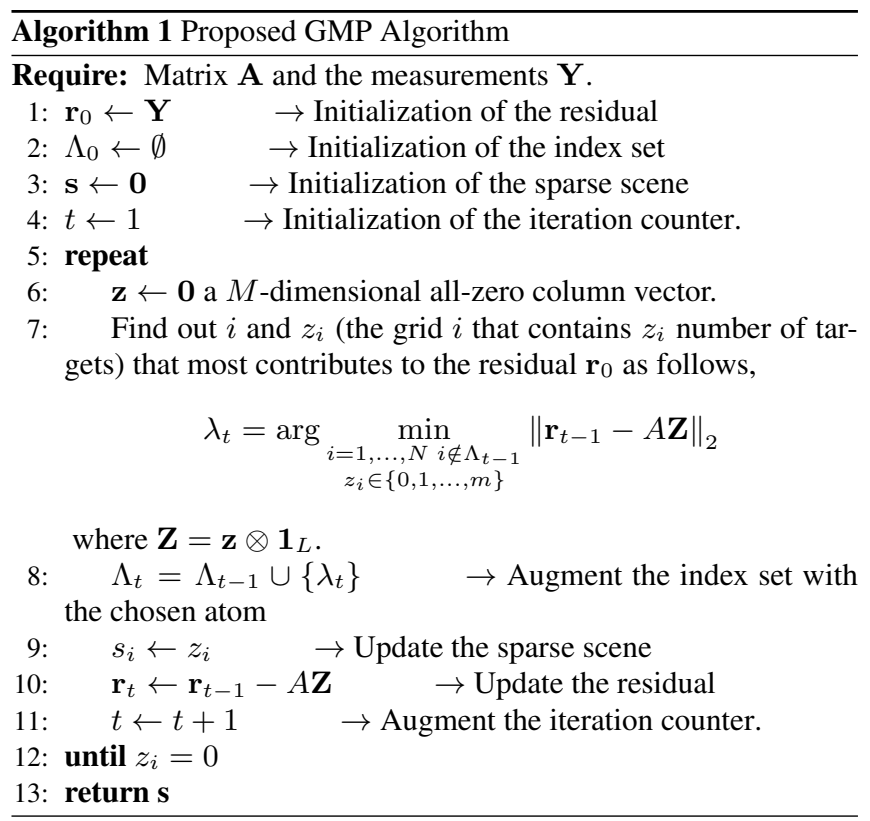

\section{NUMERICAL EVALUATION}

In this section, we evaluate the performance of the proposed approach in terms of target counting error and localization error as in [2]. A target counting error (COE) may occur either due to missdetection or double-counting and is defined as the ratio of the difference between the estimated number and actual number to the actual number of targets in the following way,

$$
\mathrm{COE}=\frac{\sum_{j=1}^{N}\left|s_{j}-\hat{s}_{j}\right|}{\sum_{j=1}^{N} s_{j}},
$$

where $\hat{s}_{j}$ denotes the estimated number of targets in the $i$-th grid.

The localization error (LOCE) is a metric that provides information on the distance between the estimated locations and the real locations, and is given by [2],

$$
\mathrm{LOCE}=\frac{\sum_{j=1}^{n_{\min }} \sqrt{\left(x_{j}-x_{j}^{\prime}\right)^{2}+\left(y_{j}-y_{j}^{\prime}\right)^{2}}}{n_{\min } \cdot r},
$$

where $\left(x_{n}, y_{n}\right)$, for $n=1, \ldots, k \cdot m$, denote the true locations of the targets; $\left(x_{\tilde{n}}^{\prime}, y_{\tilde{n}}^{\prime}\right)$, for $\widetilde{n}=1, \ldots, K$, denote the estimated locations of the targets, with $K$ being the total number of estimated targets; $n_{\min }=\min \{k \cdot m, \widetilde{n}\}$ and $r$ is the grid size, which is used to normalize the localization error. The matching between estimated locations and the real targets is performed based on the shorter distance between them.

Regarding the simulation set-up, we consider a $120 \mathrm{~m} \times 120 \mathrm{~m}$ area divided into $30 \times 30$ grid points $(N=900)$. The measurements are collected from $M$ sensors which are randomly deployed over the set of the grid center points. At each realization of the experiment, we randomly select $k$ grid points out of $N$ and put $s_{i}$ targets at each selected grid. The maximum value of $s_{i}$ is $m=3$. The channel follows a complex Gaussian distribution with a scale parameter $\sigma=$ $\sqrt{0.5}$. We set $P_{0}=1$ and $\alpha=2$.

Figure 1(a) shows an example of true target and sensor locations, with $M=40$ and $k=5$. The Signal-to-Noise Ratio (SNR) is set 


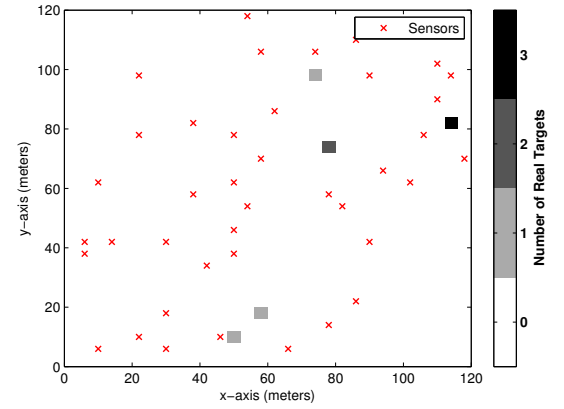

(a)

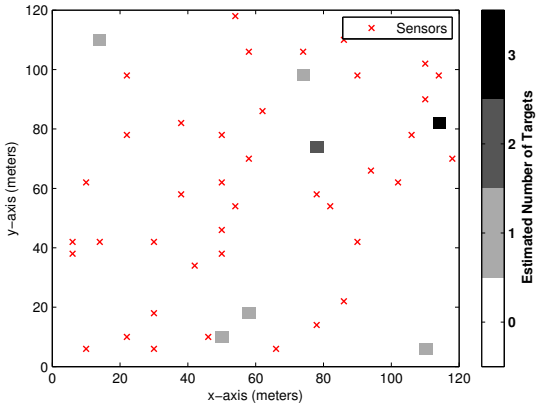

(b)

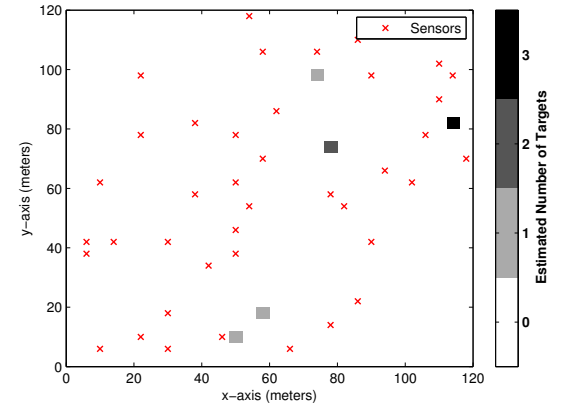

(c)

Fig. 1. Example realization with $M=40, k=5$ and $S N R=25 d B$ : (a) True target and sensor locations, (b) SMV-based technique [2], and (c) Proposed approach.
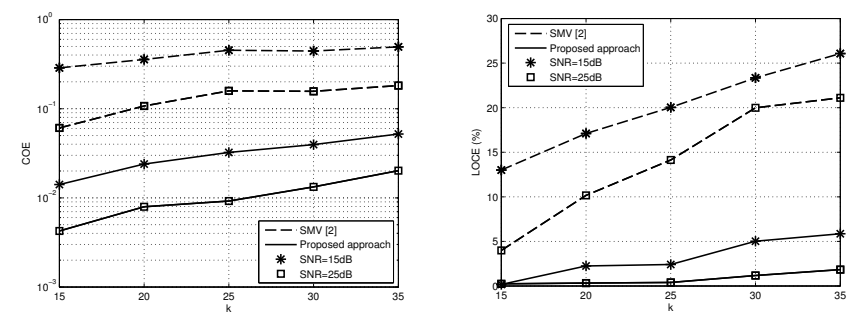

Fig. 2. Performance evaluation with respect to the sparsity level $k$ for $\mathrm{SNR}=15 \mathrm{~dB}$ and $\mathrm{SNR}=25 \mathrm{~dB}$ when $\mathrm{M}=160$. L is set to 4 for the proposed approach.

to $25 \mathrm{~dB}$ in vector $\mathbf{y}$ (4) and for each of the sensing realizations in (9). Fig. 1(b) and Fig. 1(c) depict the recovered scene when considering the SMV approach proposed in [2] and the MMV approach proposed in this paper with $L=4$, respectively. Clearly, the SMV approach generates few false targets while the proposed approach successfully recovers the scene. Therefore, the joint processing of the MMV results in better localization and counting performance.

Next figures depict the comparison between the CS-based target counting and localization technique presented in [2] and the one proposed here for 100 Monte Carlo runs. Figure 2 shows the $\mathrm{COE}$ and LOCE metrics for $\mathrm{SNR}=15 \mathrm{~dB}$ and $\mathrm{SNR}=25 \mathrm{~dB}$ when $\mathrm{M}=160$ and the sparsity level $k$ varies from 15 to 35 . Figure 2 makes evident the degradation of the both techniques when the sparsity level $k$ and the noise level increase. As predicted before, the proposed approach together with the proposed GMP algorithm achieve lower errors compared to the SMV technique proposed in [2].

Similarly, Fig. 3 presents the COE and LOCE metrics for $\mathrm{k}=10$ and $\mathrm{k}=30$ when $\mathrm{SNR}=25 \mathrm{~dB}$ and the number of sensors $M$ varies from 40 to 160. Again, Fig. 3 demonstrates the superiority of the proposed GMP algorithm. Moreover, it is observed that COE and LOCE decrease as the number of sensors $M$ increases or the sparsity level $k$ decreases.

It is worth to mention that the superiority of the proposed method with multiple measurement vectors comes at the cost of the increased observation time. However, this is not an issue while considering a very few number of snapshots of the RSS measurements in WSNs (note that, in the presented results, only 4 measurement vectors were considered at each sensor).
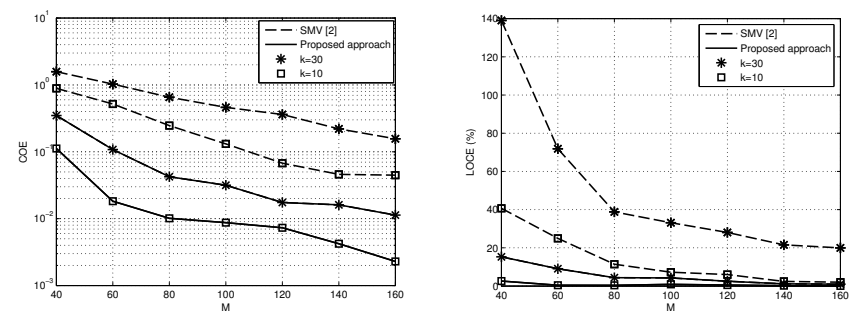

Fig. 3. Performance evaluation with respect to the number of sensors $M$ for $\mathrm{k}=10$ and $\mathrm{k}=30$ when $\mathrm{SNR}=25 \mathrm{~dB}$. $\mathrm{L}$ is set to 4 for the proposed approach.

\section{CONCLUSIONS}

This paper has introduced a novel formulation for the CS-based target counting and localization problem in WSNs considering the availability of multiple measurement vectors. Moreover, a novel GMP algorithm has been proposed which does not need the prior knowledge of sparsity level unlike the most of the existing CS-based recovery algorithms. The numerical results based on computer simulations showed the effectiveness and the superiority of the proposed technique when compared with its SMV counterpart in terms of target counting and localization accuracies. In our future work, we plan to extend the proposed MMV formulation for the case where multiple measurement vectors can be acquired both in time and spatial dimensions considering multiple antennas at each sensor, and also for the case where the sparsity level changes with time.

\section{ACKNOWLEDGEMENT}

This work was supported by FNR, Luxembourg under the CORE projects "SeMIGod" and "SATSENT". 


\section{REFERENCES}

[1] L.M. Borges, F.J. Velez, and A.S. Lebres, "Survey on the Characterization and Classification of Wireless Sensor Network Applications," IEEE Commun. Surveys Tuts., vol. 16, no. 4, pp. 1860-1890, 2014.

[2] B. Zhang, X. Cheng, N. Zhang, Y. Cui, Y. Li, and Q. Liang, "Sparse Target Counting and Localization in Sensor Networks based on Compressive Sensing," IEEE Int. Conf. on Computer Commun. (INFOCOM), Shanghai, China, pp. 22552263, Apr. 2011.

[3] S. Gezici, "A Survey on Wireless Position Estimation," SPRINGER Wireless Personal Communications, vol. 44, no. 3, pp. 263-282, Feb. 2008.

[4] J. Wang, D. Fang, X. Chen, Z. Yang, T. Xing, and L. Cai, "LCS: Compressive Sensing based Device-Free Localization for Multiple Targets in Sensor Networks," IEEE Int. Conf. on Computer Communications (INFOCOM), Turin, Italy, pp. 145-149, Apr. 2013.

[5] H. Jamali-Rad, H. Ramezani, and G. Leus, "Sparse MultiTarget Localization using Cooperative Access Points," IEEE Sensor Array and Multichannel Signal Processing Workshop (SAM), Hoboken, NJ, USA, pp. 353-356, Jun. 2012.

[6] E.J. Candes and M.B. Wakin, "An Introduction to Compressed Sampling," IEEE Signal Process. Mag., vol. 25, no. 2, pp. 2130, Mar. 2008.

[7] D.L. Donoho, “Compressed Sensing," IEEE Trans. Inf. Theory, vol. 52, no. 4, pp. 1289-1306, Apr. 2006.

[8] S.K. Sharma, M. Patwary, and M. Abdel-Maguid, "Spectral Efficient Compressive Transmission Framework for Wireless Communication Systems," IET Signal Processing, vol. 7, no. 7, pp. 558-564, Sep. 2013.

[9] E. Lagunas and M. Najar, "Spectral feature detection with sub-nyquist sampling for wideband spectrum sensing," IEEE Trans. Wireless Commun., vol. 14, no. 7, Jul. 2015.

[10] S.K. Sharma, S. Chatzinotas, and B. Ottersten, "Compressive Sparsity Order Estimation for Wideband Cognitive Radio Receiver," IEEE Trans. Signal Process., vol. 62, no. 19, pp. 49844996, Oct. 2014.

[11] J. Meng, H. Li, and Z. Han, "Sparse Event Detection in Wireless Sensor Networks using Compressive Sensing," Annual Conf. on Information Sciences and Systems (CISS), Baltimore, MD, USA, pp. 181-185, Mar. 2009.

[12] A. Oka and L. Lampe, "Distributed Target Tracking Using Signal Strength Measurements by a Wireless Sensor Network," IEEE J. Sel. Areas Commun., vol. 28, no. 7, pp. 1006-1015, Sep. 2010.

[13] C-C. Pu and W-Y. Chung, "Mitigation of Multipath Fading Effects to Improve Indoor RSSI Performance," IEEE Sensors Journal, vol. 8, no. 11, pp. 1884-1886, Nov. 2008.

[14] X. Li, "RSS-based Location Estimation with Unknown Pathloss Model," IEEE Trans. Wireless Commun., vol. 5, no. 12, pp. 3626-3633, Dec. 2006.

[15] D. Wu, D. Chen, K. Xing, and X. Cheng, "A Statistical Approach for Target Counting in Sensor-Based Surveillance Systems," IEEE Int. Conf. on Computer Communications (INFOCOM), Orlando, FL, USA, pp. 226-234, Mar. 2012.
[16] N. Shrivastava, R. Mudumbai, U. Madhow, and S. Suri, "Target Tracking with Binary Proximity Sensors," ACM Trans. on Sensor Networks, vol. 5, no. 4, pp. 1-33, Nov. 2009.

[17] Q. Le and L.M. Kaplan, "Target Tracking using Proximity Binary Sensors," IEEE Aerospace Conf., Big Sky, MT, USA, pp. 1-10, Mar. 2011.

[18] Y. Guo, B. Hua, and L. Yue, "Energy-Based Target Numeration in Wireless Sensor Networks," Future Generation Commun. and Networking (FGCN), Jeju, Korea, pp. 380-385, Dec. 2007.

[19] Q. Fang, F. Zhao, and L. Guibas, "Lightweight Sensing and Communication Protocols for Target Enumeration and Aggregation," ACM Int. Symp. on Mobile Ad-Hoc Networking and Computing (MobiHoc), Annapolis, MD, USA, pp. 165-176, Jun. 2003.

[20] M. Weiss, "Passive WLAN Radar Network Using Compressed Sensing," IET Int. Conf. in Radar Systems, Glasgow, UK, Oct. 2012.

[21] Matthias WeiB, "Target Detection with a Distributed WLAN Radar Network using Compressive Sensing," Int. Workshop on Compresed Sensing applied to Radar (CoSeRa), Bonn, Germany, May 2012.

[22] J.S. Picard and A.J. Weiss, "Localization of Multiple Emitters by Spatial Sparsity Methods in the Presence of Fading Channels," Workshop on Positioning Navigation and Communication (WPNC), Dresden, Germany, pp. 62-67, Mar. 2010.

[23] V. Cevher, M.F. Duarte, and R.G. Baraniuk, "Distributed Target Localization via Spatial Sparsity," European Signal Processing Conf. (EUSIPCO), Lausanne, Switzerland, Aug. 2008.

[24] C. Feng, S. Valaee, and Z. Tan, "Multiple Target Localization Using Compressive Sensing," IEEE Global Telecommunications Conf. (GLOBECOM), Honolulu, HI, USA, Dec. 2009.

[25] S.F. Cotter, B.D. Rao, K. Engan, and K. Kreutz-Delgado, "Sparse Solutions to Linear Inverse Problems with Multiple Measurement Vectors," IEEE Trans. Signal Process., vol. 53, no. 7, pp. 2477-2488, Jul. 2005.

[26] J. Chen and X. Huo, "Theoretical Results on Sparse Representations of Multiple-Measurement Vectors," IEEE Trans. Signal Process., vol. 54, no. 12, pp. 4634-4643, Dec. 2006.

[27] Y. Chi, "Joint Sparsity Recovery for Spectral Compressed Sensing," IEEE Int. Conf. Acoustics, Speech and Signal Processing (ICASSP), Lausanne, Switzerland, pp. 3938-3942, May 2014.

[28] J. Huang and T. Zhang, "The Benefit of Group Sparsity," Annals of Statistics, vol. 38, pp. 1978-2004, 2010.

[29] Yi. Chen, N.M. Nasrabadi, and T.D. Tran, "Simultaneous Joint Sparsity Model for Target Detection in Hyperspectral Imagery," IEEE Trans. Geosci. Remote Sens., vol. 8, no. 4, pp. 676-680, Jul. 2011.

[30] M.F. Duarte, S. Sarvotham, M.B. Wakin, D. Baron, and R.G. Baraniuk, "Joint Sparsity Models for Distributed Compressed Sensing," Workshop on Signal Processing with Adaptative Sparse Structured Representations (SPARS), Rennes, France, Nov. 2005.

[31] P. Boufounos, P. Smaragdis, and B. Raj, "Joint Sparsity Models for Wideband Array Processing," Wavelets and Sparsity XIV, SPIE Optics and Photonics, vol. 8138, pp. 4634-4643, Sep. 2011. 